\title{
ASSESSMENT OF PUBERTAL GROWTH SPURT IN EGYPTIAN ADOLESCENTS USING MIDDLE PHALANX OF THE MIDDLE FINGER (MP3) IN ALEXANDRIA GOVERNORATE: A CROSS SECTIONAL STUDY
}

\author{
Mohamed Zahir Almolki *, Fatma Abdou El-Sayed"* and Manal Yehia Fouda **
}

\begin{abstract}
Aim of this study: to assess the pubertal growth spurt in a group of Alexandria school male adolescents, using the developmental stages of the middle phalanx of the middle finger (MP3), and to evaluate the skeletal maturity age differences between Alexandria male adolescents and other populations.
\end{abstract}

Materials \& Methods: A sample of 1100 male students for Alexandria was included in this study with age range from 11 to 16 years was collected. A Radiograph for the middle phalanx of the middle finger by digital dental radiograph for the assessment of MP3 maturation stages was taken for each subject.

Results: The MP3-G stage (signifying the peak of pubertal growth spurt) was found with high prevalence in subjects aged $>13-14$ years with mean of age $13.76 \mathrm{y}$.

Conclusion: 1. The mean skeletal maturity age (MP3-G stage) that represents the peak of height velocity (PHV) in Egyptian adolescent males of Alexandria was found to be $13.76 \mathrm{y} \pm 1.15$ years. 2 The newly digital radiography of the MP3 region is definitely an available, simple technique of high clarity, low radiation.

\section{INTRODUCTION}

Growth is the result of biological processes by means of which living matter normally gets larger and it is not uniform throughout life. Adolescence is a period during which the rate of growth acceleration reaches a peak velocity and then decelerates until adulthood is achieved. There are marked individual variations in the initiation, duration, rate and amount of growth during this period of life ${ }^{1}$.

One of the objectives of orthodontic treatment during adolescence, in cases with skeletal discrepancies, is to take advantage of patient's

\footnotetext{
* M.D.Sc, Cairo University, Egypt

** Prof. of Orthodontics, Faculty of Oral and Dental Medicine, Cairo University
} 
growth changes. Maturational status can have a considerable influence on diagnosis, treatment goals, treatment planning and the eventual outcome of orthodontic treatment.

The maturity status of a child is best estimated relative to specific stages of physiologic maturity than chronological age, being not a reliable indicator. Physiological age is estimated by the maturation of one or more tissue systems. It could also be estimated by somatic, sexual, skeletal and dental maturity ${ }^{2}$ peak height velocity (PHV.

Skeletal maturity is frequently determined by assessing the maturational status and level of ossification of bony markers within the skeletal system. Among the various developmental indicators, skeletal age is considered the most reliable method to correlate with the general biological and physiological body maturation ${ }^{3,4}$.

In orthodontics, the skeletal maturity has been assessed using either hand-wrist maturation index (HWMI) as performed by Fishman ${ }^{5}$, which is one of the most reliable and valid maturation indicators, or cervical vertebrae maturation index (CVMI) as performed by Hassel $^{6}$ which is reliable and valid as Fishman's in addition to its availability. Lately, the middle phalanx of the third finger (MP3) was used by Hagg and Taranger ${ }^{3}$ and modified by Rajagopale and Kansal $^{7}$ using periapical dental radiographic films. This last maturity indicator, has the advantages of being costless, available in all Egyptian governorates dental clinics, of low radiation dose, free of superimpositions of bones or vertebrae and reliable as confirmed by many authors ${ }^{7-11}$.

Since assessment of pubertal growth spurt within the Egyptian population is lacking, a research project was carried out between the Ministry of Health and Faculty of Oral and Dental Medicine, Cairo University, represented by orthodontic department for the assessment of pubertal growth spurt using (MP3) growth stages among Egyptian adolescent males. This study was carried out in Alexandria .

\section{MATERIALS AND METHODS}

A sample of 1100 male students for Alexandria was included in this study. A Radiograph for the middle phalanx of the middle finger by digital dental radiograph for the assessment of MP3 maturation stages was taken for each subject. The subjects were divided into five groups according to their age that cover the circumpubertal period.

\section{Inclusion Criteria}

- Egyptian in origin.

- Adolescent males with an age range of 11-16 years.

\section{Exclusion Criteria}

- Endocrinal disorders.

- Systemic disease or syndromes.

- Trauma in the area of the left middle finger.

- Malnutrition.

The following records were taken for each subject:

\section{Personal Information Chart:}

Personal data including name, age, date of birth, gender and phone number were recorded for every subject. Parents and subjects were informed about the research plan, the safety and the amount of radiation exposure related to MP3, and an informed consent was signed.

\section{Radiographing of MP3:}

\section{A) Digital Radiography of MP3:}

Each subject was radiographed using digital dental radiography technique ${ }^{12}$ of high clarity and good contrast for his MP3 (middle phalanx of $3^{\text {rd }}$ left finger). For accuracy and reliability of the method, all X-rays were taken using the same machine, at the 
same distances and intensity. The X-ray was taken with a Digital Dental Portable X- Ray Machine System* and a sensor.

Each subject was instructed to place his left hand with the palm downward on a flat table. The finger concerned was aligned with the long axis of the intra-oral dental sensor, in such a way that the area to be radiographed was located in the center of the sensor.

The cone of the dental X-ray machine was positioned in light contact with the middle phalanx of the middle finger and perpendicular to the sensor (figure 1). The exposure time was 0.05 second.

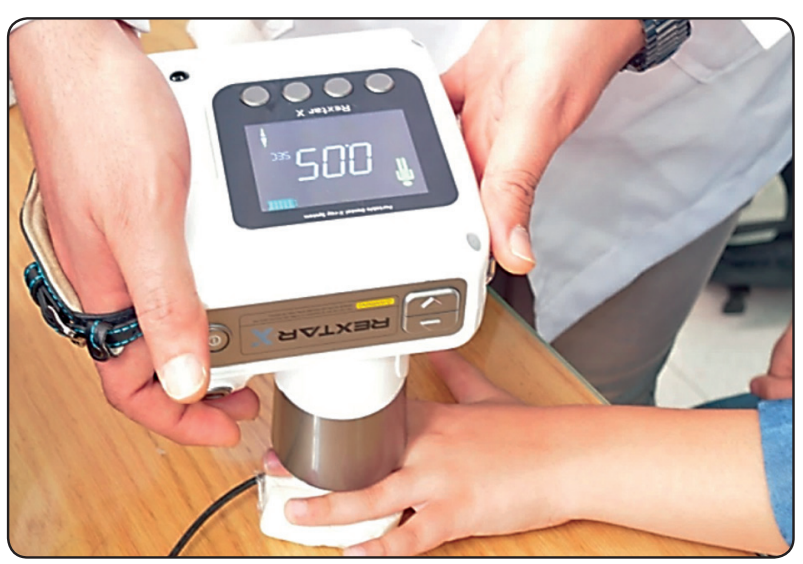

Fig. (1) The position of the dental sensor, finger.

\section{B) Assessment of MP3 Maturation Stages:}

The developmental stage of the middle phalanx of the third finger (MP3) was evaluated for each digital radiograph according to the method described by Rajagopal and Kansal (2002) ${ }^{7}$.

\section{C) Reliability of the Method:}

Intra and inter-observer reliability were assessed by evaluation of MP3 radiographs for $10 \%$ of the sample size (110), by the same observer twice at 2 occasions with two-week interval and by another observer. The intra and inter observer differences were evaluated statistically for significance. Insignificant differences confirmed reliability of the method

\section{RESULTS}

The present study was conducted on 1100 male subjects. Their age range was $11-16 \mathrm{y}$, with mean age value $13.63 \pm 1.49$ years. They showed that 162 subjects (14.7\%) were at the age group 11-12 years, 215 subjects $(19.5 \%)$ were at the age group $>12-13$ years, 205 subjects $(18.6 \%)$ were at age group $>13$ 14 years, 206 subjects $(18.7 \%)$ were at the age group $>14-15$ years, and 312 subjects $(28.4 \%)$ were at the age group $>15-16$.

There was a statistically significant difference between mean age values at different growth stages, $\mathrm{P}<0.001$, (Figure 2). Pair-wise comparisons between the stages revealed that stage (I) showed the statistically significantly highest mean age, while stage (F) showed the statistically significantly lowest mean age value. The pubertal spurt age at G-stage was $13.76 \mathrm{y}$.

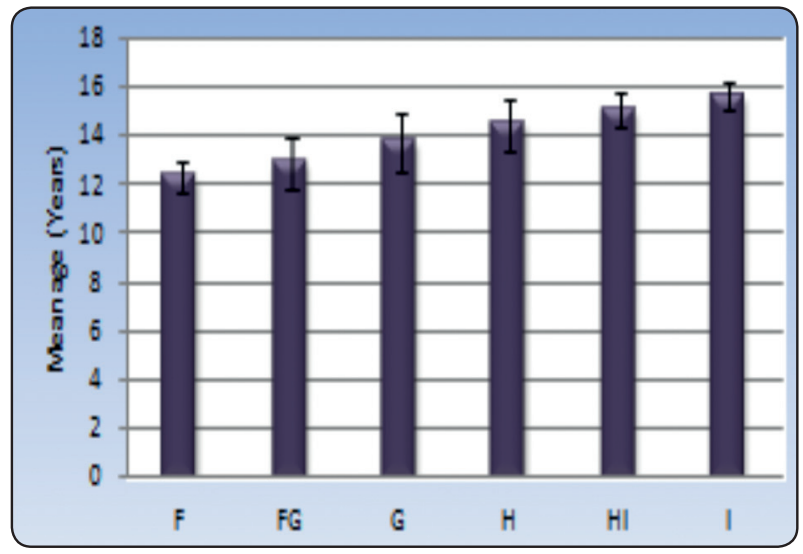

Fig. (2) Bar chart representing mean and standard deviation of age values at different growth stages.

* REXTAR-X-Dental Portable X-Ray System from Posdion, South Korea. 


\section{DISCUSSION}

The percentage distribution of the subjects enrolled in each age group was evaluated. The highest number of subjects 312 and percentage (28.4\%) was the $>15-16$ years group, while the smallest number and percentage of subjects 162 (14.7\%) was the 11-12 years group.

The percentage distribution of each of the six MP3 developmental stages in the studied sample was evaluated. The highest number 241 and percentage (21.9\%) showed MP3-FG developmental stage, followed by MP3-G stage (226, 20.5\%), followed by MP3-F stage (192, 17.5\%), followed by MP3-H stage $(172,15.6 \%)$, followed by MP3-HI stage $(160,14.5 \%)$, and the smallest number of subjects showed MP3-I stage $(109,9.9 \%)$.

In this study, the $M P 3-G$ stage which corresponds to CS-3 of CVMI (signifying the peak of pubertal growth spurt or PHV) was found with high prevalence in subjects aged $>13-14$ years with mean of age $13.76 \mathrm{y}$.

Orthopedic intervention using functional appliances that modify the condylar growth as the Activator should be started just before the pubertal growth spurt, CS-3 stage or MP3-G stage. Delaying the treatment will lead to loss of precious active growth period. On the other hand, orthopedic appliances used in maxillary protraction and expansion through modifying sutural growth, as the Facemask and expansion screws, should be started at the per-pubertal spurt; CS-2 stage or MP3FG stage ${ }^{14}$. Condylar growth spurt coincides with skeletal spurt while sutural growth spurt is earlier and coincides with the pre-peak stage ${ }^{15}$.

\section{CONCLUSIONS}

Based on the findings of this study, the following conclusions can be drawn:

1. The mean skeletal maturity age (MP3-G stage) that represents the peak of height velocity in Egyptian adolescent males of Alexandria was found to be $13.76 \mathrm{y} \pm 1.15$ years.

2. The newly digital radiography of the MP3 region is definitely an available, simple technique of high clarity, low radiation. It is also costeffective and time-saving for the assessment of the skeletal developmental stage, which is essential in diagnosis and treatment planning for orthodontists, pedodontists, as well as many other health disciplines.

\section{REFERENCES}

1. Silveira AM, Fishman LS, Subtelny JD, Kassebaum DK. Facial growth during adolescence in early, average and late maturers. Angle Orthod. 1992;62(3):185-190.

2. Demirjian A, Buschang PH, Tanguay R, Patterson DK. Interrelationships among measures of somatic, skeletal, dental, and sexual maturity. Am J Orthod. 1985;88(5):433438 .

3. Hägg U, Taranger J. Maturation indicators and the pubertal growth spurt. Am J Orthod. 1982;82(4):299-309.

4. Grave KC, Brown T. Skeletal ossification and the adolescent growth spurt. Am J Orthod. 1976; 69(6):611-619.

5. Fishman LS. Chronological versus skeletal age, an evaluation of craniofacial growth. Angle Orthod. 1979; 49(3):181-189.

6. Hassel B, Farman AG. Skeletal maturation evaluation using cervical vertebrae. Am J Orthod Dentofac Orthop. 1995;107(1):58-66.

7. Rajagopal R, Kansal S. A comparison of modified MP3 stages and the cervical vertebrae as growth indicators. J Clin Orthod. 2002;36(7):398-406.

8. Neetu Dabla VSRGAKCKLP. A Comparative Evaluation of Modified MP3and CVMI Stages As Maturation Indicators. J Indian Orthod Soc. 2006;39(3):147-154.

9. Abdel-Kader HM. The reliability of dental x-ray film in assessment of MP3 stages of the pubertal growth spurt. Am J Orthod Dentofacial Orthop. 1998;114(4):427-429.

10. Negi KS. Reliability of Mp3 (Middle Phalanx of $3 \mathrm{Rd}$ 
Finger ) Stages in Assessment of Skeletal Maturation - A Correlative. 2009;3.

11. Prasad M, Ganji VKSK, George SA, Talapaneni AK, Shetty SK. A comparison between cervical vertebrae and modified MP3 stages for the assessment of skeletal maturity. J Nat Sci Biol Med. 2013;4(1):74-80.

12. Abdel-Kader HM. The potential of digital dental radiography in recording the adductor sesamoid and the
MP3 stages. Br J Orthod. 1999;26(4):291-294.

13. Baccetti T, Franchi L, McNamara JA. The Cervical Vertebral Maturation (CVM) Method for the Assessment of Optimal Treatment Timing in Dentofacial Orthopedics. Semin Orthod. 2005;11(3):119-129.

14. Baccetti T, Franchi L, Toth LR, McNamara JA. Treatment timing for Twin-block therapy. Am J Orthod Dentofacial Orthop. 2000;118(2):159-170. 